Research Article

\title{
On the Effects of Viscosity on the Shock Waves for a Hydrodynamical Case_-Part I: Basic Mechanism
}

\author{
Huseyin Cavus \\ Physics Department, Arts \& Science Faculty, Canakkale Onsekiz Mart University, 17100 Canakkale, Turkey \\ Correspondence should be addressed to Huseyin Cavus; h_cavus@comu.edu.tr
}

Received 19 June 2013; Revised 31 October 2013; Accepted 31 October 2013

Academic Editor: Gregory Laughlin

Copyright (C) 2013 Huseyin Cavus. This is an open access article distributed under the Creative Commons Attribution License, which permits unrestricted use, distribution, and reproduction in any medium, provided the original work is properly cited.

\begin{abstract}
The interaction of shock waves with viscosity is one of the central problems in the supersonic regime of compressible fluid flow. In this work, numerical solutions of unmagnetised fluid equations, with the viscous stress tensor, are investigated for a onedimensional shock wave. In the algorithm developed the viscous stress terms are expressed in terms of the relevant Reynolds number. The algorithm concentrated on the compression rate, the entropy change, pressures, and Mach number ratios across the shock wave. The behaviour of solutions is obtained for the Reynolds and Mach numbers defining the medium and shock wave in the supersonic limits.
\end{abstract}

\section{Introduction}

Shock waves are rather common phenomena in the supersonic flows of any fluid. They arise in many areas that are related with hydrodynamics such as fluid mechanics, aerodynamics, astrophysics, solar physics, and space physics. If a medium is shocked, particles behind the shock front experience both compressive and shear forces. They push the particles away from their original equilibrium positions.

Shock waves are studied by many authors. Somow and Spector [1] studied the basic mechanisms of the hydrodynamic shocks in the solar atmosphere during flares. Effects of inhomogeneities in solar wind plasma on the interplanetary shock waves are also studied by Heinemann [2]. Their observational studies are given in the work of De Lucas et al. [3]. Magara and Shibata [4] worked on the formation of shock waves due to plasma ejections in the solar atmosphere. Khidr and Mahmoud [5] obtained results for the case of an arbitrary Prandtl number in strong shock waves, using a modified power law for viscosity in terms of temperature and Mach number. Kuznetsov [6] studied the stabilities of shock waves in hydrodynamic discontinuities and flows in the relaxation zone. On the other hand, Hamad and El-Fayes [7] studied the entropy change for the structure of inviscid plasma by neglecting viscosity in the gas phase. Compression rate in bow shocks was studied by Kabin [8], and the Euler equations for a one-dimensional hydrodynamic model were considered [9]. In a more recent study, Swift et al. [10] derived expressions for shock formation, based on the local curvature of the flow characteristics during dynamic compression.

Motion occurs in a continuous medium because of some external causes like pressure gradients and body forces; this way, the fluid velocity distribution becomes inhomogeneous in general. In consequence, resistance to variations in the distribution of cohesive forces in fluids may such come into play to remove inhomogeneities in velocities. Such resistive effects produce the phenomenon of viscosity in fluid motions [11]. Therefore, viscosity, which measures the resistance of a fluid system as a function of velocity, should also be taken into account as one of the most important effects in the equations of motion. For the shock heating of solar corona, Orta et al. [12] obtained that the shock thickness and profile depend on the viscosity and resistivity, and heating ultimately occurs because of them. Dispersive shock waves were studied by Ballai et al. [13]. They showed that dispersion will alter the amplitude and propagation speed of a shock wave.

For supersonic flows the coexistence of shock waves with viscous effects produces interesting features. The interaction of viscous effects with a shock wave is a key feature in many fluid dynamics systems [14]. Considerable theoretical and 
numerical interest should be expressed in the strengthening of a shock wave propagating through a viscous medium. The viscous interactions of a solar wind stream were studied by Korzhov et al. [15] and it was found that the Kelvin-Helmholtz instability is excited due to the presence of shear flows. Coronal mass ejections (CMEs) and solar wind are the main results of the solar activity. These events can drive interplanetary shock waves and produce geomagnetic storms. Study of these events is very important for space weather purposes. The shock waves occur where the solar wind changes from being supersonic (with respect to the surrounding interplanetary medium) to being subsonic. In the supersonic regime of compressible gas flow, the interaction of shock waves with viscosity is one of the central problems. To define such type of shock process, the Navier-Stokes equations should be solved. Mathematically this process can be approximated to a hydrodynamical case.

The Reynolds number is another defining feature of fluid motion. Reynolds, a British scientist, showed that the transition from laminar to turbulent flow is directly related to a dimensionless number defined as the Reynolds number [16]. This dimensionless number gives the relation between inertial and viscous forces in a fluid flow. If the inertial forces are large (the flow rate is high), then the flow will rather occur turbulently. When the viscous forces are large enough compared to the inertial ones, then a laminar fluid flow character will be observed.

The main purpose of this paper is to describe the basic mechanism of the shock wave problem, in which the viscous terms cannot be neglected in deriving the jump relations. To define this type of shock process, the Navier-Stokes equations defining the momentum conservation should be solved. The viscous behaviour of a fluid can be described by using the Reynolds number. The formalism used for a one-dimensional study is explained in more detail in the following section. Solutions are presented in terms of physical parameters such as compression rate, Mach number and pressure ratios, and entropy change across the shock waves. Results will be given in Section 3. Our results are compared with other similar works in Section 4, together with a discussion and conclusion.

\section{Physical Formulation of the Problem}

2.1. Basic Equations. The basic formulation for unmagnetised plasma is also known as the system of hydrodynamical equations in conservative form. For a compressible viscous shock wave for steady flow, the equations, are [17-19]

$$
\begin{gathered}
\int_{A} \rho u_{j} n_{j} d A=0, \\
\int_{A}\left(\rho u_{i} u_{j}+p \delta_{i j}-\tau_{i j}\right) n_{j} d A=0, \\
\int_{A}\left(\rho H-\tau_{i j}\right) u_{j} n_{j} d A=0, \\
p=\rho \mathrm{RT} .
\end{gathered}
$$

These basic equations to be solved consist of the conservation of the mass (1), the momentum (2), and the energy for adiabatic flow (3) together with the ideal gas law (4). Here $\rho, u, d A, n_{i}, \delta_{i j}, \tau_{i j}, p$, and $H$ denote the density, velocity, infinitesimal surface area, unit normal $(i=1,2,3)$, the Kronecker delta, viscous stress tensor, pressure, and the total enthalpy, respectively.

2.2. One-Dimensional Problem. Using these steady viscous flow relations with letting subscript 1 and 2 as the upstream and downstream shock front, respectively, fluid equations can be written for a one-dimensional case (say along $x$-axes). The Rankine-Hugoniot jump relations [20] can be obtained through (1)-(3). We use the viscous tensor in one dimension as

$$
\tau=\tau_{x x}=\frac{4}{3} \mu \frac{d u}{d x},
$$

where $\mu$ is the dynamic viscosity coefficient. We define the compression ratio, $\kappa$, in terms of the upstream and downstream velocities:

$$
\frac{u_{2}}{u_{1}}=\kappa^{-1} .
$$

We can define the Reynolds number, Re, through

$$
\mu\left(\frac{d u}{d x}\right) \approx \frac{\left(\rho u^{2}\right)}{\operatorname{Re}} .
$$

With the use of (5)-(7) in (1)-(4), we can obtain the equation as follows (after a little algebra):

$$
\begin{aligned}
& {\left[\left(\frac{1}{2}-\frac{4}{3} \frac{1}{\mathrm{Re}_{1}}\right)(\gamma-1) M_{1}^{2}+1\right] \kappa^{2}} \\
& \quad-\left[\left(1-\frac{4}{3} \frac{1}{\mathrm{Re}_{1}}\right) \gamma M_{1}^{2}+1\right] \kappa+\left(\frac{\gamma+1}{2}-\frac{4}{3} \frac{1}{\mathrm{Re}_{2}}\right) M_{1}^{2}=0 .
\end{aligned}
$$

Here $\mathrm{Re}_{1}$ and $\mathrm{Re}_{2}$ are the upstream and downstream Reynolds number, respectively, and $M_{1}$ is the value of the upstream Mach number. The last equation is also called the general equation and it can be solved numerically by imposing the appropriate conditions. Since $1<\gamma<2$, this equation has just one positive root. Moreover the solution reduces to the inviscid hydrodynamic value for the vanishing values of dynamic viscosity.

2.3. Value of the Downstream Reynolds Number. In order to simplify the problem, the downstream Reynolds number, $\mathrm{Re}_{2}$, can be expressed as a function of the adiabatic index, $\mathrm{Re}_{1}$, and $M_{1}$ is similar to Bruhn et al. [21]. Figure 1 shows the change of $\mathrm{Re}_{2}$ with respect to $\mathrm{Re}_{1}$ for different values of $M_{1}$ for the monatomic with gas $\gamma=5 / 3$. There is a limitation on the upstream Mach number $\left(M_{1}\right)$ in order to have a shock wave. Shock waves are common phenomena in supersonic upstream flows $\left(M_{1}>1\right)$ of any fluid. From the quadratic structure of (8) and the limitation on the value 


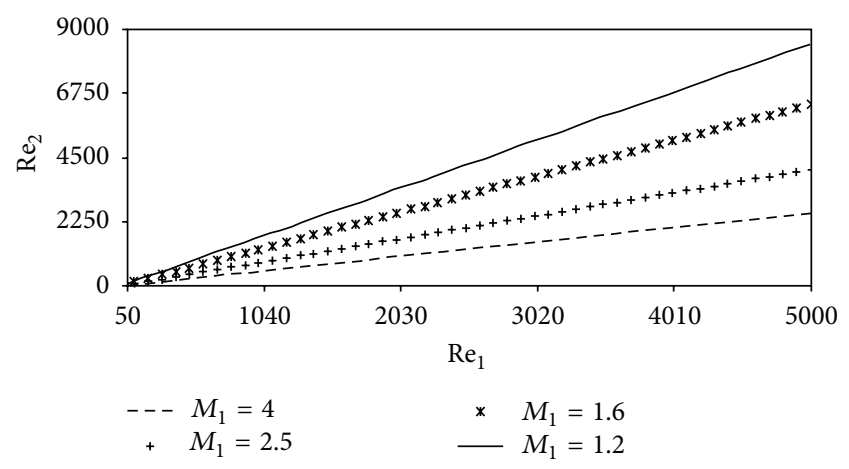

FIGURE 1: Behaviour of the $\operatorname{Re}_{2}$ as a function of $\mathrm{Re}_{1}$ for different values of $M_{1}$.

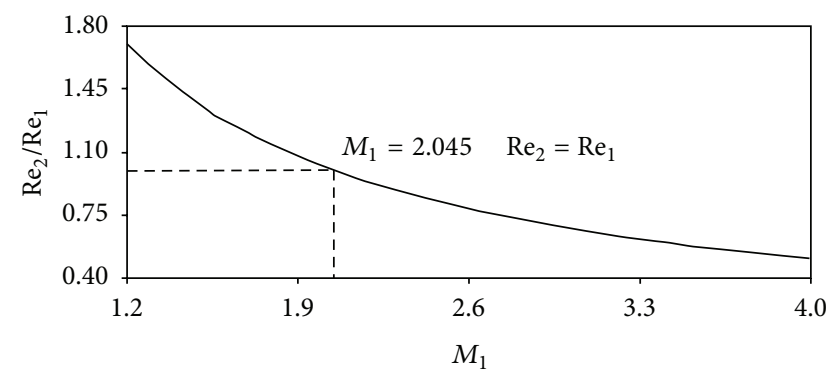

Figure 2: Upstream Mach number, $M_{1}$, dependence of $\operatorname{Re}_{2} / \operatorname{Re}_{1}$. Note that, at $M_{1} \approx 2, \operatorname{Re}_{2}=\operatorname{Re}_{1}$.

of $M_{1}, \mathrm{Re}_{1}$ must be greater than or equal to 50 as shown in the horizontal axis of Figure $1 . \mathrm{Re}_{2}$ increases with respect to increasing values of $\mathrm{Re}_{1}$. On the other hand, $\mathrm{Re}_{2}$ has larger values for smaller values of $M_{1}$. In Figure 2, the ratio $\mathrm{Re}_{2} / \mathrm{Re}_{1}$ is given as a function of $M_{1}$. It is decreasing as the upstream Mach number increases and the ratio of the Reynolds number is equal to unity (i.e., $\mathrm{Re}_{1}=\mathrm{Re}_{2}$ ) at the value of $M_{1} \approx 2$ as shown in this figure. This value is considered as the transition point from weak to strong shocks.

\section{Model Results}

We have attempted to find some special solutions of (8) and the Rankine-Hugoniot jump relations using the method given in the previous section with an algorithm developed and adapted to Maple 9.5. Table 1 represents the variation of the parameters describing the physical structure for the inviscid shock waves. These parameters are compression rate (i.e., densities ratio), the Mach numbers ratio $\left(M_{2} / M_{1}\right)$, pressures ratio $\left(p_{2} / p_{1}\right)$, and the entropy difference $\left(S_{2}-S_{1}\right)$ provided by the solutions of inviscid forms of the hydrodynamic equations. These values will be used as a reference model. Finally, one can easily conclude that vanishing values of the viscosity means higher values of Reynolds number from its definition.
TABLE 1: Change of physical parameters for inviscid shock waves $(\mu=0$, i.e., higher Re).

\begin{tabular}{lcccc}
\hline$M_{1}$ & $\kappa$ & $M_{2} / M_{1}$ & $p_{2} / p_{1}$ & $S_{2}-S_{1}$ \\
\hline 1.200 & 1.297 & 0.705 & 1.550 & 0.055 \\
1.900 & 2.185 & 0.328 & 4.263 & 1.839 \\
2.600 & 2.770 & 0.210 & 8.200 & 5.061 \\
3.300 & 3.136 & 0.154 & 13.363 & 8.574 \\
4.000 & 3.368 & 0.123 & 19.750 & 11.961 \\
\hline
\end{tabular}

TABLE 2: Representation of pressures ratio $\left(p_{2} / p_{1}\right)$ with respect to $M_{1}$ for $\operatorname{Re}_{1}=50$ and $\operatorname{Re}_{1}=4500$.

\begin{tabular}{ccc}
\hline$M_{1}$ & $\mathrm{Re}_{1}=50$ & $\mathrm{Re}_{1}=4500$ \\
\hline 1.2 & 1.446 & 1.549 \\
1.9 & 4.192 & 4.262 \\
2.6 & 8.117 & 8.199 \\
3.3 & 13.281 & 13.362 \\
4.0 & 19.700 & 19.750 \\
\hline
\end{tabular}

3.1. Variations of Parameters with respect to Mach Numbers. As explained in the previous section, distributions of physical parameters can be obtained from the solutions of (8) and the Rankine-Hugoniot relations. The related quantities, Mach numbers and pressures ratios, compression rate, and entropy change are presented in Figures 3-7 and Table 2 for a monatomic gas with $\gamma=5 / 3$.

Figures 3 and 4 show the changes in the compression rate $(\kappa)$ with respect to the upstream Mach number, $M_{1}$, and $\mathrm{Re}_{2} / \mathrm{Re}_{1}$, respectively. Figure 3 was drawn for two different values of $\mathrm{Re}_{1}$. It shows that, for weak shocks $\left(M_{1}<2\right)$, there is no variation with respect to upstream Mach number in accordance with Khidr and Mahmoud [5]. However, for strong shocks $\left(M_{1}>2\right)$, there are considerable differences between the values of compression rate for different values of $\mathrm{Re}_{1}$. Another important result of strong shocks is the fact that the value of $\kappa$ approaches its inviscid value (3.368) as given in Table 1 for the higher values of $\operatorname{Re}_{1}$ (e.g., 4500) shown as the upper dashed line in Figure 3.

Figure 4 represents the $\mathrm{Re}_{2} / \mathrm{Re}_{1}$ dependence of $\kappa$. It tends to decrease with increasing values of ratio. It also exhibits an interesting feature. For the small values of the Reynolds numbers ratio, it gives a larger compression rate at the smaller value of $\mathrm{Re}_{1}$ (i.e., 50). It has comparable values in the vicinity of the $\mathrm{Re}_{2} / \mathrm{Re}_{1}=1$ for both high and low values of $\mathrm{Re}_{1}$. However, for the larger values of ratio, the shape is visibly changed, and it gives larger compression rates at the larger value of $\mathrm{Re}_{1}=4500$.

Table 2 summarizes the results obtained for pressures ratio $\left(p_{2} / p_{1}\right)$ with the values of the upstream Mach number in the supersonic region for two different values of $\operatorname{Re}_{1} \cdot p_{2} / p_{1}$ approaches its inviscid value $\left(p_{2} / p_{1}=19.750\right)$ given in Table 1 for the higher values of $\mathrm{Re}_{1}$ in the strong shock regime.

In Figure 5, variation of $M_{2} / M_{1}$ is presented as a function $\mathrm{Re}_{2} / \mathrm{Re}_{1}$ for two different values of the upstream Reynolds number. It has the tendency of growing with the 
TABLE 3: Reynolds number, $\operatorname{Re}_{1}$, dependence of $M_{2} / M_{1}$ and $p_{2} / p_{1}$ for different values of $M_{1}$ and $\operatorname{Re}_{2} / \operatorname{Re}_{1}$ values.

\begin{tabular}{lcccccccc}
\hline \multirow{2}{*}{$\operatorname{Re}_{1}$} & \multicolumn{2}{c}{$M_{1}=1.2\left(\mathrm{Re}_{2} / \mathrm{Re}_{1}=1.704\right)$} & \multicolumn{2}{c}{$M_{1}=1.6\left(\mathrm{Re}_{2} / \mathrm{Re}_{1}=1.278\right)$} & \multicolumn{2}{c}{$M_{1}=2.5\left(\mathrm{Re}_{2} / \mathrm{Re}_{1}=0.818\right)$} & \multicolumn{2}{c}{$M_{1}=4\left(\mathrm{Re}_{2} / \mathrm{Re}_{1}=0.511\right)$} \\
& $M_{2} / M_{1}$ & $p_{2} / p_{1}$ & $M_{2} / M_{1}$ & $p_{2} / p_{1}$ & $M_{2} / M_{1}$ & $p_{2} / p_{1}$ & $M_{2} / M_{1}$ & $p_{2} / p_{1}$ \\
\hline 100 & 0.7224 & 1.5017 & 0.4315 & 2.9166 & 0.2207 & 7.5220 & 0.1217 & 19.7281 \\
1000 & 0.7068 & 1.5454 & 0.4293 & 2.9467 & 0.2211 & 7.5585 & 0.1225 & 19.7481 \\
2000 & 0.7060 & 1.5477 & 0.4291 & 2.9483 & 0.2212 & 7.5605 & 0.1226 & 19.7491 \\
3000 & 0.7057 & 1.5485 & 0.4291 & 2.9489 & 0.2212 & 7.5612 & 0.1226 & 19.7494 \\
4000 & 0.7056 & 1.5489 & 0.4291 & 2.9492 & 0.2212 & 7.5615 & 0.1226 & 19.7495 \\
5000 & 0.7055 & 1.5491 & 0.4291 & 2.9493 & 0.2212 & 7.5617 & 0.1226 & 19.7496 \\
\hline
\end{tabular}

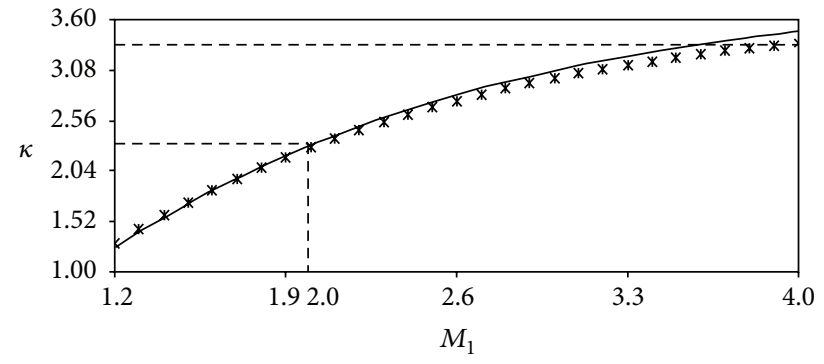

* $\operatorname{Re}_{1}=4500$

- $\operatorname{Re}_{1}=50$

FIGURE 3: Variations of compression rate with respect to $M_{1}$ for different values of upstream Re.

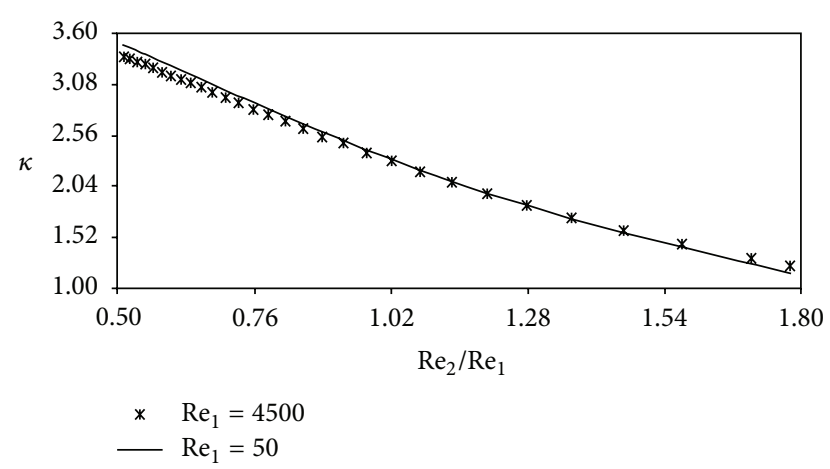

FIGURE 4: Variations of compression rate with respect to $\operatorname{Re}_{2} / \operatorname{Re}_{1}$.

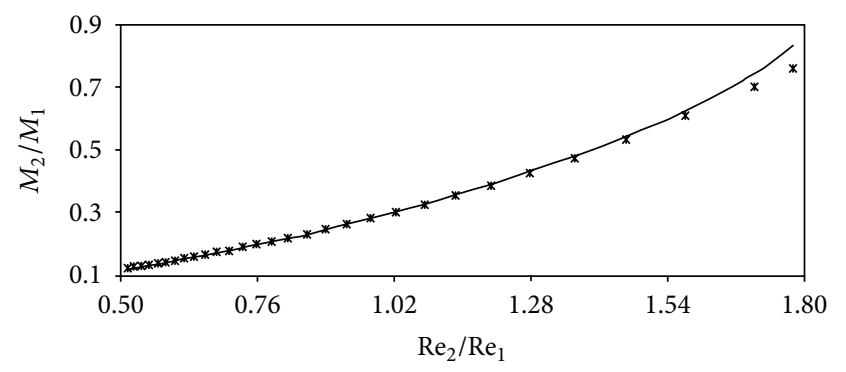

* $\begin{aligned} \mathrm{Re}_{1} & =4500 \\ \mathrm{Re} & =50\end{aligned}$

FIGURE 5: Change of Mach numbers ratio $\left(M_{2} / M_{1}\right)$ as a function of $\mathrm{Re}_{2} / \mathrm{Re}_{1}$ for two different $\mathrm{Re}_{1}$ values.

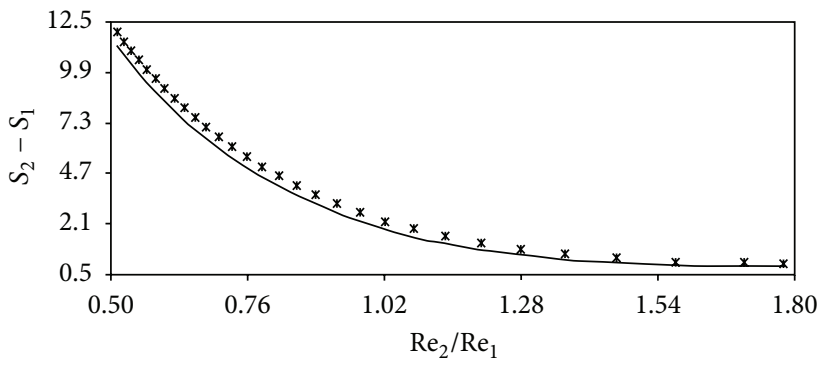

* $\mathrm{Re}_{1}=4500$

- $\operatorname{Re}_{1}=50$

FIGURE 6: Distributions of entropy difference $S_{2}-S_{1}$ as a function of $\mathrm{Re}_{2} / \mathrm{Re}_{1}$ for two different values of $\mathrm{Re}_{1}$.

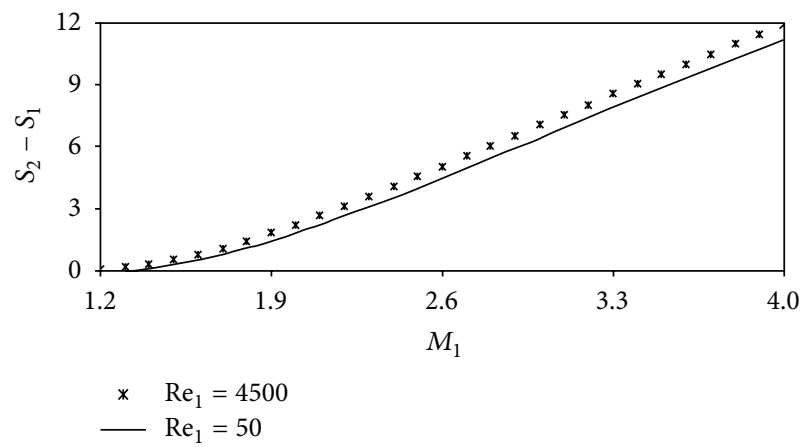

Figure 7: Same as Figure 6, but as a function of $M_{1}$.

values of $\mathrm{Re}_{2} / \mathrm{Re}_{1}$. This parameter shows another important consequence. The shock wave becomes a Prandtl-Meyer expansion wave (i.e., $M_{2}>M_{1}$ ) for very high values of $\mathrm{Re}_{2} / \mathrm{Re}_{1}$.

The entropy change depicted in Figures 6 and 7 can be expressed in terms of pressures ratio and compression rate as follows [22]:

$$
S_{2}-S_{1}=\frac{R}{\gamma-1} \ln \left[\frac{p_{2}}{p_{1}} \kappa^{-\gamma}\right] .
$$

In Figure 6, variation of $S_{2}-S_{1}$ with respect to $\mathrm{Re}_{2} /$ $\operatorname{Re}_{1}$ ratio has the tendency to decrease with increasing ratio. For higher values of $\mathrm{Re}_{1}, S_{2}-S_{1}$ has larger values as expected since higher values of the Reynolds number close the case of inviscid flow value given in Table 1. 


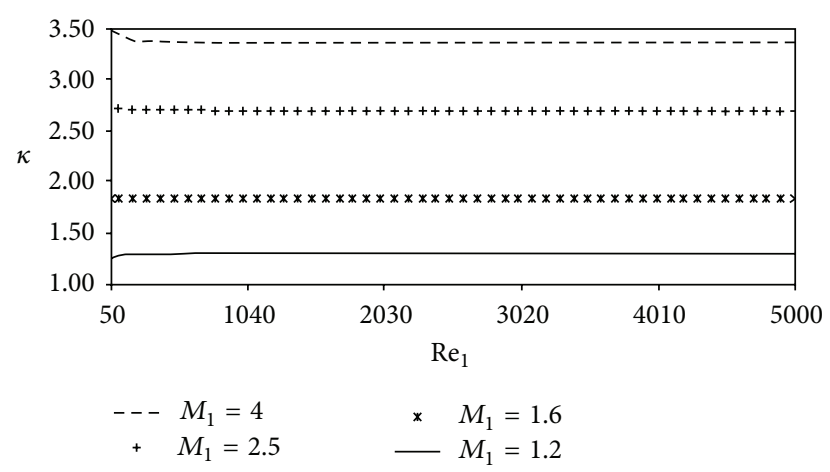

FIGURE 8: Variations of compression rate as a function of $\mathrm{Re}_{1}$ at four different upstream Mach number values.

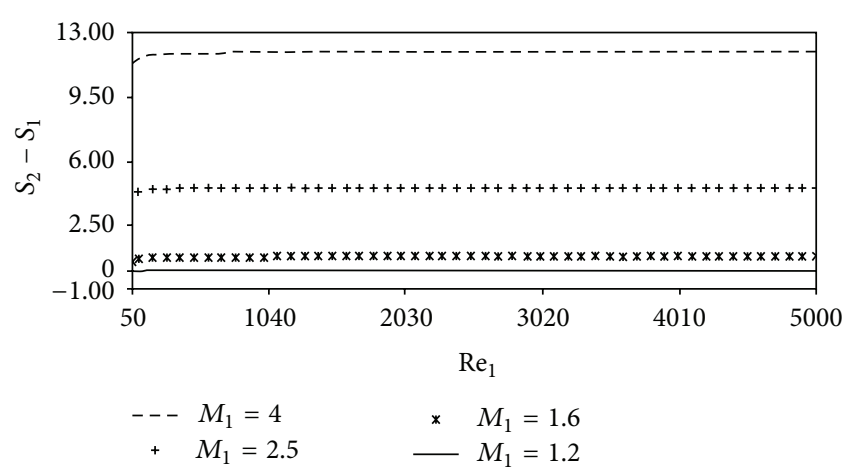

FIGURE 9: Variations of entropy difference $S_{2}-S_{1}$ with $\operatorname{Re}_{1}$ for four different values of $M_{1}$.

In Figure 7, dependence of entropy change, $S_{2}-S_{1}$, with respect to $M_{1}$ is given. It has the tendency to increase with increasing $M_{1}$. For the higher values of $\operatorname{Re}_{1}$ (i.e., vanishing values of viscosity), $S_{2}-S_{1}$ has larger values as expected.

3.2. Variations with Respect to Reynolds Number. In this subsection, the distributions of physical parameters are given as a function of the upstream Reynolds number, $\mathrm{Re}_{1}$, in Figures 8 and 9 and Table 3 again for a monatomic gas.

In Figure 8, the values of compression rate $(\kappa)$ are given as a function of $\operatorname{Re}_{1}$ for different values of $M_{1}$. It is increasing with the values of the upstream Mach number and also reaches very high values for strong shock cases of $M_{1}=$ 2.5 and 4.

From Figure 9, it is seen that the entropy change is negative for smaller values of $M_{1} \leq 1.2$ in the region of very small $\operatorname{Re}_{1}$ (i.e., $\left.<50\right)$ across the shock. This case violates the second law of thermodynamics (i.e., $S_{2}-S_{1}<0$ ). Thus the weak shock waves (i.e., $M_{1}=1.2$ ) are nearly isentropic.

In Table 3, $\mathrm{Re}_{1}$ dependencies of the Mach numbers and pressures ratios are given for different values of $M_{1}$. One of the most important features of these results is that the values of $M_{2} / M_{1}$ have a decreasing tendency with the increasing values of $\mathrm{Re}_{1}$ for $\mathrm{Re}_{2} / \mathrm{Re}_{1}>1$ and a decreasing tendency for $\mathrm{Re}_{2} / \mathrm{Re}_{1}<1$. The critical value of the Mach number for this turning point was found to be 2.045 which equates the
Reynolds numbers ratio to unity as in Figure 2. This point is important not only for the Reynolds numbers ratio but also for the strength of shock waves. The pressures ratio $\left(p_{2} / p_{1}\right)$ has the increasing tendency and reaches a value close to the inviscid flow value given in Table 1 for the higher values of the Reynolds number with increasing values of Mach number (i.e., strong shocks).

\section{Discussion and Conclusion}

When a fluid is shocked, particles after the shock front experience both compressive and stress forces. In such a movement viscous forces largely depend on plasma particles motions. In the present work, the detailed analysis of the structure of steady hydrodynamical equations including the viscous stress tensor in conservative forms was attempted in order to investigate the effects of the Reynolds number on the shock waves.

Since the Reynolds number gives the relation between inertial and viscous forces of the fluid flow, the character of flow can be determined from the Reynolds number. If inertial forces are dominant and the Reynolds number Re $>2000$, flow is turbulent. If viscous forces surpass, then the value of the Reynolds number $\mathrm{Re}<2000$ and flow becomes laminar. Using fluid-velocity dependence, viscosity can change the character of flow. As expected, a high Reynolds number can be a result of vanishing viscosity and its lower values are the result of higher viscosity. Since the viscous stress is written as a function of fluid velocity, the viscosity can be determined by the change in the fluid velocity. Such a dissipative mechanism controls the values of flow variables. The viscous terms in the Rankine-Hugoniot jump relations can be expressed in terms of the Reynolds number using its definition. In this present paper, the basic mechanism of the problem is given in order to investigate the effects of viscosity and the Reynolds number. The behaviour of shock waves in supersonic flows with varying Reynolds and Mach numbers was examined using analytical and numerical methods.

We obtained that, in strong shocks, physical parameters have the increasing tendency to reach a value close to the inviscid flow values obtained for the higher values of the upstream Reynolds number making the flow turbulent. Depending on increasing values of viscosity, the values parameters decrease across the shock.

As in Mace and Adamson Jr. [23], for the shock waves in a transonic region, compression rate is independent of the Reynolds number. The results in this regime agree well with the inviscid values. These results are in good concordance with the results obtained in the work of Kabin [8] for weak shocks. Viscosity makes the profiles of compression ratio smooth for very high values of the Mach number. The compression rate is increasing with the values of the upstream Mach number. However, there are considerable differences between the compression rates for different values of $\mathrm{Re}_{1}$ for strong shocks. That is, compression rate is higher for laminar upstream flow and is lower for turbulent upstream flow.

The Mach numbers ratio has a decreasing tendency for the larger values of $\mathrm{Re}_{1}$ in the region of $\mathrm{Re}_{2} / \mathrm{Re}_{1}>1$. 
In other words, a shock wave becomes a Prandtl-Meyer expansion wave for very high values of $\mathrm{Re}_{2} / \mathrm{Re}_{1}$ for the transonic values of the upstream Mach number $(<2)$ as it is seen from Figure 1. $M_{2} / M_{1}$ decreases for $\mathrm{Re}_{2} / \mathrm{Re}_{1}<$ 1 in the region of $M_{1}>2$. When the upstream Reynolds number is dominant, the shock waves become very strong (see Figure 2). Viscosity makes the problem smooth, and the downstream shock waves become more turbulent if $\mathrm{Re}_{1}$ is turbulent in the transonic limits of $M_{1}$ in accordance with the results of Jamme et al. [24]

As given in the work of Gamba [25], for the turbulent case of $\operatorname{Re}_{1}$ (i.e., >2000), we obtained that the entropy change increases with increasing values of upstream shock speed. It is also clear that the entropy change decreases with the increasing values of the Reynolds numbers ratio $\mathrm{Re}_{2} / \mathrm{Re}_{1}$ and it has close results for both extreme values of the $\mathrm{Re}_{1}$. It is also found that the weak shocks are nearly isentropic for smaller values of upstream Re similar to the results of De Sterck et al. [26].

In summary, the present study provides results useful for future studies including dissipative shocks. These types of shock waves arise frequently in the cases where the velocity of fluid is greater than the local sound speed; they find an application in gas dynamics, fluid mechanics, aerodynamics, astrophysics, solar physics, and space physics, for both magnetised and unmagnetised fluid motions.

It is planned to use the present algorithm and its results to be applied to solar coronal shock waves. The effects of viscous flows on the shock wave happened after 13/12/2006CME is under study in order to apply the present results. The simulations of the effects of the Reynolds number on the shock waves for magnetized plasma in both one and multidimensions are also under study.

\section{Acknowledgment}

The author acknowledges with thanks technical and computing support from the Research Foundation of the Canakkale Onsekiz Mart University (Project no. 2011/012).

\section{References}

[1] B. V. Somow and A. R. Spector, "Hydrodynamic shock wave formation in the solar chromosphere and corona during flares," Space Science Reviews, vol. 32, no. 1-2, pp. 27-41, 1982.

[2] M. Heinemann, "Effects of solar wind inhomogeneities on transit times of interplanetary shock waves," Journal of Atmospheric and Solar-Terrestrial Physics, vol. 64, no. 3, pp. 315-325, 2002.

[3] A. de Lucas, R. Schwenn, A. Dal Lago, E. Marsch, and A. L. Clúa de Gonzalez, "Interplanetary shock wave extent in the inner heliosphere as observed by multiple spacecraft," Journal of Atmospheric and Solar-Terrestrial Physics, vol. 73, no. 10, pp. 1281-1292, 2011.

[4] T. Magara and K. Shibata, "Plasma ejections and shock waves in the solar atmosphere," Journal of Atmospheric and SolarTerrestrial Physics, vol. 70, no. 2-4, pp. 546-554, 2008.

[5] M. A. Khidr and M. A. A. Mahmoud, "The shock-wave structure for arbitrary Prandtl numbers and high mach numbers," Astrophysics and Space Science, vol. 113, no. 2, pp. 289-301, 1985.
[6] N. M. Kuznetsov, "Stability of shock waves," Soviet Physics Uspekhi, vol. 32, no. 11, article 993, 1989.

[7] H. Hamad and F. El-Fayes, "Analytical solution for shock waves in dusty gases," Acta Mechanica, vol. 119, pp. 53-63, 1996.

[8] K. Kabin, "A note on the compression ratio in MHD shocks," Journal of Plasma Physics, vol. 66, no. 4, pp. 259-274, 2001.

[9] C. Escudero, "On one-dimensional models for hydrodynamics," Physica D, vol. 217, no. 1, pp. 58-63, 2006.

[10] D. C. Swift, R. G. Kraus, E. N. Loomis, D. G. Hicks, J. M. McNaney, and R. P. Johnson, "Shock formation and the ideal shape of ramp compression waves," Physical Review E, vol. 78, no. 6, Article ID 066115, 2008.

[11] Z. U. A. Warsi, Fluid Dynamics: Theoretical and Computational Approaches, CRC Press, Boca Raton, Fla, USA, 1999.

[12] J. A. Orta, M. A. Huerta, and G. C. Boynton, "Magnetohydrodynamic shock heating of the solar corona," The Astrophysical Journal Letters, vol. 596, no. 1, pp. 646-655, 2003.

[13] I. Ballai, E. Forgacs-Dajka, and A. Marcu, "Dispersive shock waves in the solar wind," Astronomische Nachrichten, vol. 328, no. 8, pp. 734-737, 2007.

[14] J. Blazek, Computational Fluid Dynamics: Principles and Applications, Elsevier, Amsterdam, The Netherlands, 2001.

[15] N. P. Korzhov, V. V. Mishin, and V. M. Tomozov, "On the viscous interaction of solar wind streams," Soviet Astronomy, vol. 29, pp. 215-218, 1985.

[16] O. Reynolds, "An experimental investigation of the circumstances which determine whether the motion of water shall be direct or sinuous, and of the law of resistance in parallel channels," Philosophical Transactions of the Royal Society, vol. 174, pp. 935-982, 1883.

[17] L. D. Landau and E. M. Lifshitz, Fluid Mechanics, Pergamon Press, New York, NY, USA, 1959.

[18] D. D. Knight, The Handbook of Fluid Dynamics, CRC Press, Boca Raton, Fla, USA, 1998.

[19] A. R. Choudhuri, The Physics of Fluids and Plasmas, Cambridge University Press, Cambridge, Mass, USA, 1998.

[20] Y. B. Zel'dovich and Y. P. Raizer, Physics of Shock Waves and High-Temperature Hydrodynamic Phenomena, Dover, New York, NY, USA, 2002.

[21] F. C. Bruhn, K. Pauly, and V. Kaznov, "Extremely low mass spherical rovers for extreme environments and planetary exploration enabled with MEMS," in Proceedings of the 8th International Symposium on Artificial Intelligence, Robotics and Automation in Space (i-SAIRAS '05), Munich, Germany, 2005.

[22] F. M. White, Fluid Mechanics, McGraw-Hill, New York, NY, USA, 1994.

[23] J. L. Mace and T. C. Adamson Jr., "Shock waves in transonic channel flows at moderate Reynolds number," AIAA Journal, vol. 24, no. 4, pp. 591-598, 1986.

[24] S. Jamme, J.-B. Cazalbou, F. Torres, and P. Chassaing, "Direct numerical simulation of the interaction between a shock wave and various types of isotropic turbulence," Flow, Turbulence and Combustion, vol. 68, no. 3, pp. 227-268, 2002.

[25] I. M. Gamba, "Boundary-layer formation for viscosity approximations in transonic flow," Physics of Fluids A, vol. 4, no. 3, pp. 486-490, 1992.

[26] H. de Sterck, H. Deconinck, S. Poedts, and D. Roose, “ A bow shock flow containing (almost) all types of ("exotic") MHD discontinu," in Proceedings of the 7th International Conference on Hyperbolic Problems Theory, Numerics, Applications, M. Fey and R. Jeltsch, Eds., Birkhäuser, Basel, Switzerland, 1998. 

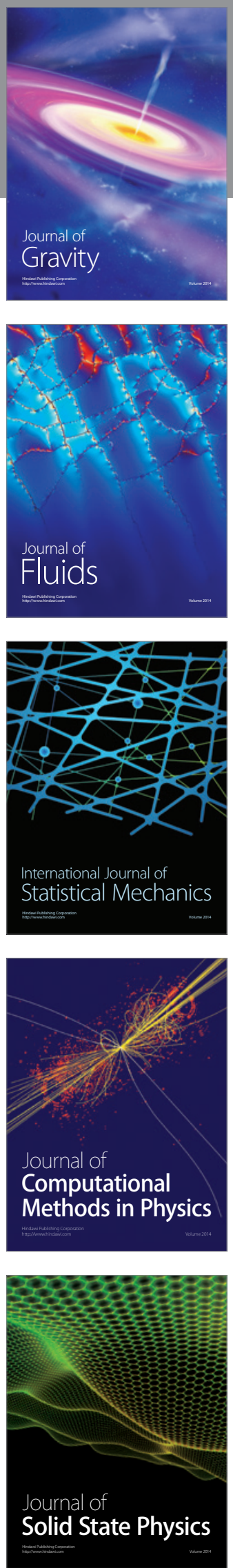

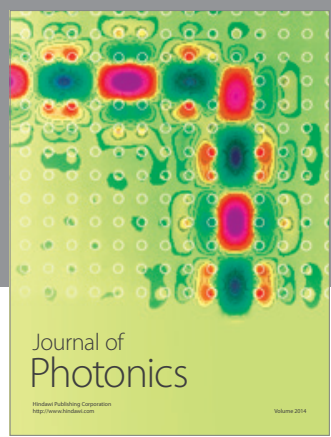

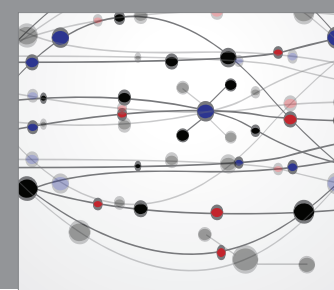

The Scientific World Journal

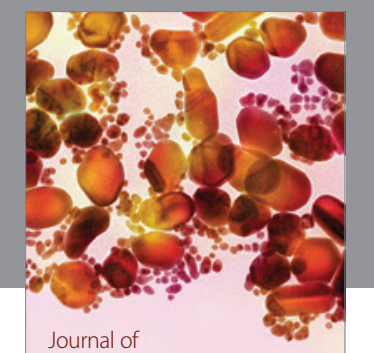

Soft Matter
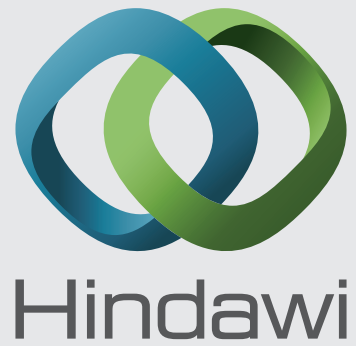

Submit your manuscripts at

http://www.hindawi.com
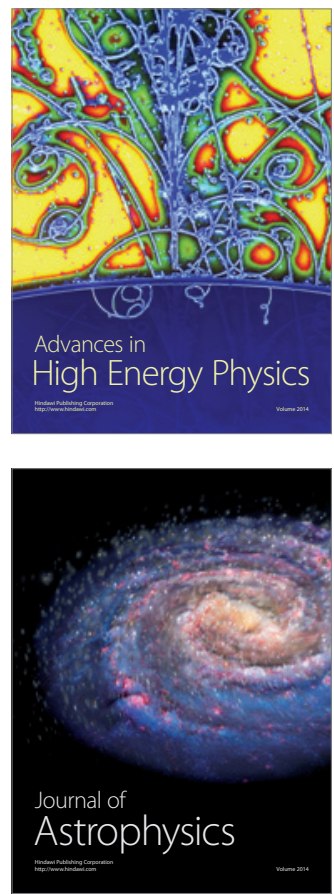
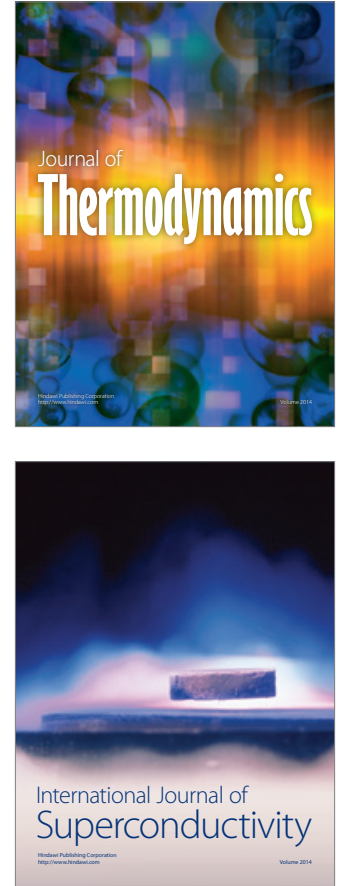
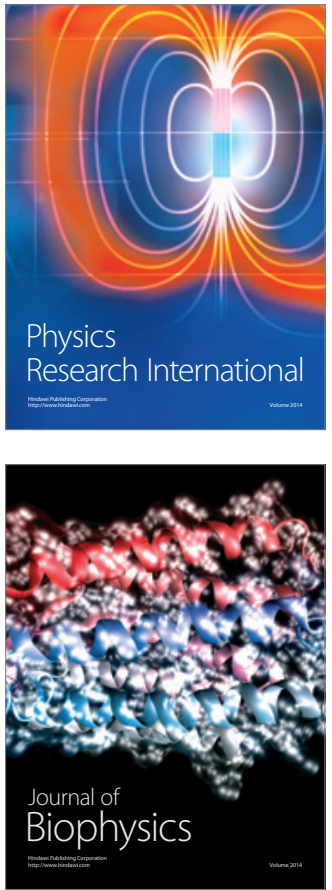
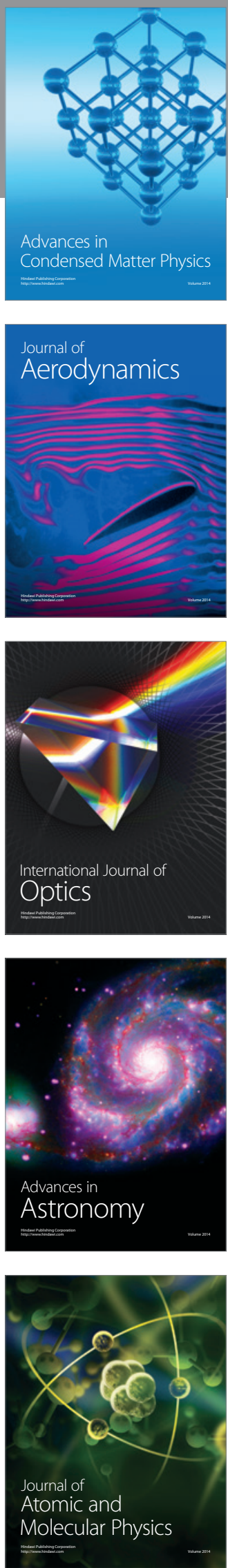